\title{
Machine Learning Techniques for Filtering of Unwanted Messages
}

\author{
J. Hari Purushotham \\ M.Tech (CSE) \\ KHIT,Guntur, \\ India
}

\author{
B. Tarakeswara Rao, PhD \\ Professor (Dept. of CSE) \\ KHIT, Guntur, \\ India
}

\author{
B. Sathyanarayana Reddy \\ Associate Professor (Dept.of CSE) \\ KHIT, Guntur \\ India
}

\begin{abstract}
Online Social Networking in these days is most powerful way to share the Information, thought, event and many more. In the Definition of technology we usually follow the Industry of Information Technology where, we describe the high-level contributions of this paper and discuss potential future research directions. Despite the massive popularity of online social networks, surprisingly little is known about how people are using them to connect and share content. To better understand the structure of online social networks, in this paper we conducted a large-scale measurement study that collected data on the social networks of four popular sites, covering over 12 million users and 400 million links, in order to remove the unwanted messages. Machine Learning Text Categorization is also used to categorize the short text messages.
\end{abstract}

\section{Keywords}

Online social networks, information filtering, short text classification, policy-based personalization.

\section{INTRODUCTION}

Most normal intuitive medium to convey is online social network. A few sorts of data or substance will be shared between the clients, the kind of substance are sound, video, pictures and so on. As the Amount of substance will be extremely limitless data sifting is utilized. OSN give less measure of security in posting undesirable messages. Data sifting is utilized for disconnected reason. Capacity of a client to naturally control the messages composed on the client all, by sifting extra correspondence will be termed as data separating [1]. Machine learning content arrangement strategy is additionally utilized as a part of proposed, to consequently allot the short content in light of the substance [3]. Methods incorporate a few stages, short content classifier is one of the stride it incorporates content representation, machine learning based order, spiral premise capacity system. Second step is of sifting principles and blacklist administration. Sifting rules comprises of maker detail and separating guideline .Finally, Blacklist is incorporated. In proposed Blacklist standard is actualized. Mechanized framework called sifted divider is assessed, which is utilized to channel undesirable messages from client divider. Content based message separating is bolstered in proposed framework which is not upheld to existing framework. Two level orders are performed. Short messages are classifies as Neutral and Non impartial in first level. Unbiased messages are ordered in second stage. Aside from characterization, effective tenet is abused called sifting rules. Sifting rules give the consequence of ML order process, which channel the client divider and relationship of client. Further Blacklist is likewise upheld by the framework; it can be said as clients who post the undesirable message will be kept in blacklist for specific timeframe. By utilizing this guideline, OSN is given more security. This paper is propelled by unsecure of OSN. Our framework, BL system used to maintain a strategic distance from messages from undesired makers, autonomous from the substance.

\section{RELATED WORK}

In the related work we are going to discuss the recent methods over the content-based filtering in Online Social Networking (OSN). Macro Vanetti, Elena Ferrari, and Moreno Carullo [1] In this paper provides the user to have a straight rule over their own private wall to avoid the unwanted messages. The main aim of this paper is, users have a straight control over messages posted on their own private space. So we are using the automated system called Filtered wall (FW), which have a capacity to filter unwanted messages .This system will blocks only the unwanted messages send by the user. Drawback of this paper is user will not be blocked; only the content posted by L. Roy and R.J.Mooney [13] uses mutual filtering method, but in our proposed system content based filtering is used. It explains the content based book proposal system that develops information pulling out and machine learning technique for text categorization. B. Carminative, M.vanetti, E.ferrari, M.Craullo [7] In this paper the system can generally take decision about the message which is blocked, dude to the acceptance depends up on statistical information. Bodicev and M.Sokolova [5] classification of text put in complex and specific terminology; need the application of learning process. Fractional Matching method is applied which shrink the text for confining the text characteristic. Fractional matching develops a language model. The output of fractional matching compression provides consistent care of text classification J.Colbeck [6] Social network is the common concentration group in network. To make the faith many explanations are required. Two level approaches are stated to combine gloss, trust and origin. We state an algorithm for concluding trust relationship with origin information and trust gloss in web social network. Film trust application is introduced which uses trust to movie ranking and ordering the review. We consider film trust give the good crop. M.Carullo, E.Binaghi, and I. Gallo [8] clustering of document is helpful in many field. Two categories of clustering general purpose and text tilting, these both will be used for clustering of information. Novel heuristic online document clustering is predictable, which is proficient in clustering of text tilting parallel measures. Presentation measure is done in F-measure, and then it will be counterpart up with other methods. The result will indicate the power of proposed system. The user will block content based message filtering and short text classification support by this system.

\section{EXISTING SYSTEM}

In our Existing system, Machine Learning text categorization techniques is used, which routinely allocate with each short text message, which categories based on its content. A system 
called Filtered Wall (FW) is exploited, which is used to filter the unwanted messages. A significant rule called filtering rules which is used to decide which content should be displayed on user wall. This system will not be more secure. The main problem of this paper is, it will block only the unwanted message, but the user who sends that message will not be blocked.

\section{PROPOSED SYSTEM}

In this paper we proposed large scale estimation ponder that gathered information on the interpersonal organizations of four well known locals, covering more than 12 million clients and 400 million connections, with a specific end goal to uproot the undesirable messages. Machine Learning Text Categorization is additionally used to sort the short instant messages.

\section{AUTOMATIC TEXT CLASSIFICATION}

Today, text classification is a need because of the expansive measure of content Documents that we need to manage day by day. Text Classification is the assignment to group records into predefined classes. Text Classification is likewise called content order, record arrangement, and Document classification. There are two methodologies for classification manual classification and automatic classification. Significant innovations for Text Classification are
1. Text Clustering
2. Information Retrieval (IR)
3. Information Filtering
4. Information Extraction (IE)
5. Text Classification

Text clustering that Create groups of archives with no outside data. Information Retrieval (IR) that recover an arrangement of records important to an inquiry. Information Filtering Filter out superfluous archives through collaborations. Information Extraction (IE) removes pieces of data, e.g., individual names, dates, and places, in records. In text Classification there is no inquiry, no communications, no outer data, it just choose themes of Documents. Text Classification use in different fields like E-mail spam sifting, arrange daily paper articles and newswires into themes, compose Web pages into various leveled classes, sort diaries and edited compositions by subject classifications (e.g., MEDLINE, and so on.), relegating universal clinical codes to quiet clinical records and so forth.

Established techniques used for text classification work well on datasets with large documents such as newswires corpora [6], but suffer when the documents in the corpus are short. There are various representation techniques and neural learning strategy that can be use combine to semantically categorize short texts. For classifying text or short texts a hierarchical strategy is use [5]. A different sets of features has been consider for text categorization ,there are three different issues pertaining namely document representation, classifier construction, and classifier evaluation. Text categorization is the activity of labeling natural language texts with thematic categories from a predefined set [4]. However the most appropriate feature set and feature representation are Bag of Words( BOW), Document properties (DP) and Contextual Features (CF).[7][3]

\subsection{BAG-of-Words Model (BOW)}

The bag of-words model is a rearranging representation utilized as a part of characteristic dialect handling and information retrieval (IR). In this model, a content, (for example, a sentence or a Document) is spoken to as the bag (multi set) of its words, ignoring language structure and even word arrange however keeping variety. As of late, the bag ofwords model has additionally been utilized for PC vision. The bag of-words model is regularly utilized as a part of techniques for Document order, where the (recurrence of) event of every word is utilized as an element for preparing a classifier.

\subsection{Documents Properties (DP)}

Document properties as a uniform premise for cooperation. Document properties express abnormal state elements of Documents that are significant to clients and usable by frameworks. Document properties are straightforwardly connected with archives, as opposed to with Document stockpiling areas. This implies records will hold properties notwithstanding when moved starting with one place then onto the next, and that property task can have a fine granularity.

\subsection{Contextual Features (CF)}

A contextualized procedure may permit IR frameworks to learn and foresee what data a searcher needs, figure out how and when data ought to be shown, present results relating them to past data and to the errands the client has been occupied with and choose who else ought to get the new data [10].

\subsection{Vector Space Model (VSM)}

The Vector space model or term vector model is a mathematical model for speaking to content records (and any articles, when all is said in done) as vectors of identifiers, for example, for instance, file terms. It is utilized as a part of data separating, data recovery, indexing and pertinence rankings. The VSM representation of the content that portrays the earth where messages are posted (subjects of the talk, name of the gathering or whatever other important content encompassing the messages) [9].

\subsection{Machine Learning-Based Classification}

There are two methodologies that you can take that is rule based approach and machine learning-based methodology. Rule based methodology compose an arrangement of principles that order reports. Machine learning-based methodology utilizing an arrangement of test reports that are grouped into the classes (preparing information) naturally make classifiers taking into account the preparation information. Machine Learning is another method for inspiring PCs to arrange archives. Machine learning is typically not managed based. Rather, it is regularly measurably based. It's the capacity of a machine to enhance its execution taking into account past results so; machine learning archive order is the capacity of a machine to enhance its report characterization execution in light of past aftereffects of record grouping.

\section{FILTERING RULES AND BLACKLIST MANAGEMENT}

Filtering Rules:

The same message on OSNs may have different meanings and relevance based on who writes it. It is necessary to apply constraints on messages. Constraints can be selected on several different criteria's. User can state what contents should 
be blocked or displayed on filtered wall by means of Filtering rules. Filtering rules are specified on the basis of user profile as well as user social relationship.FR is dependent on following factors
1. Author
2. Creator Spec
3. Content Spec
4. Action

An author is a person who defines the rules. Creator Spec denotes the set of OSN user and Content Spec is a Boolean expression defined on content. Action denotes the action to be performed by the system on the messages matching content Spec and created by users identified by creator Spec [9].

The same message on OSNs might have distinctive implications and importance in view of who composes it. It is important to apply imperatives on messages. Imperatives can be chosen on a few diverse criteria's. Client can state what substance ought to be blocked or showed on sifted divider by method for Filtering rules. Filtering rules are determined on the premise of client profile and also client social relationship.FR is subject to taking after variables
1. Author
2. Creator Spec
3. Content Spec
4. Action

An author is a man who characterizes the tenets. Creator Spec means the arrangement of OSN client and Content Spec is a Boolean expression characterized on substance. Action signifies the activity to be performed by the framework on the messages coordinating content Spec and made by clients recognized by creator Spec [9].

\subsection{Online Setup Assistant for FRS Thresholds}

There are issues of setting limits to channel guideline. Online Setup Assistant (OSA) method permits client to choose an arrangement of messages from dataset of messages. Such messages are chosen by certain measure of non unbiased messages taken from a small amount of the dataset and not fitting in with the preparation/test sets, are characterized by the ML to have, for every message, the second level class participation esteem. On the premise of this the client advises the framework the choice to acknowledge or dismiss the message. The accumulation and handling of client choices on an adequate arrangement of messages circled over every one of the classes permits to figure modified edges speaking to the client demeanor in tolerating or dismissing certain substance.

\subsection{Blacklist}

BL clients are those clients whose messages are kept autonomous from their substance. BL rules empower the divider proprietor to decide clients to be hindered on the premise of their profiles and association with divider proprietor. This banning should be possible for a predefined period or everlastingly agreeing divider proprietor's craving. Like FR, BL is additionally reliant on author, creator specification and creator behavior. For denoting users' bad behavior considered two main measures. The first is, if any client has as of now into BL and again embedded commonly in it for given time interim, say more noteworthy than a given limit, he/she may should stay in the BL.

\subsection{Filtered Wall Architecture}

The architecture of OSN services is three-tire structure of three layers (Fig1).

These three layers are

- Social Network Manager(SNM)

- $\quad$ Social Network Application(SNA)

- $\quad$ Graphical User Interface(GUI)

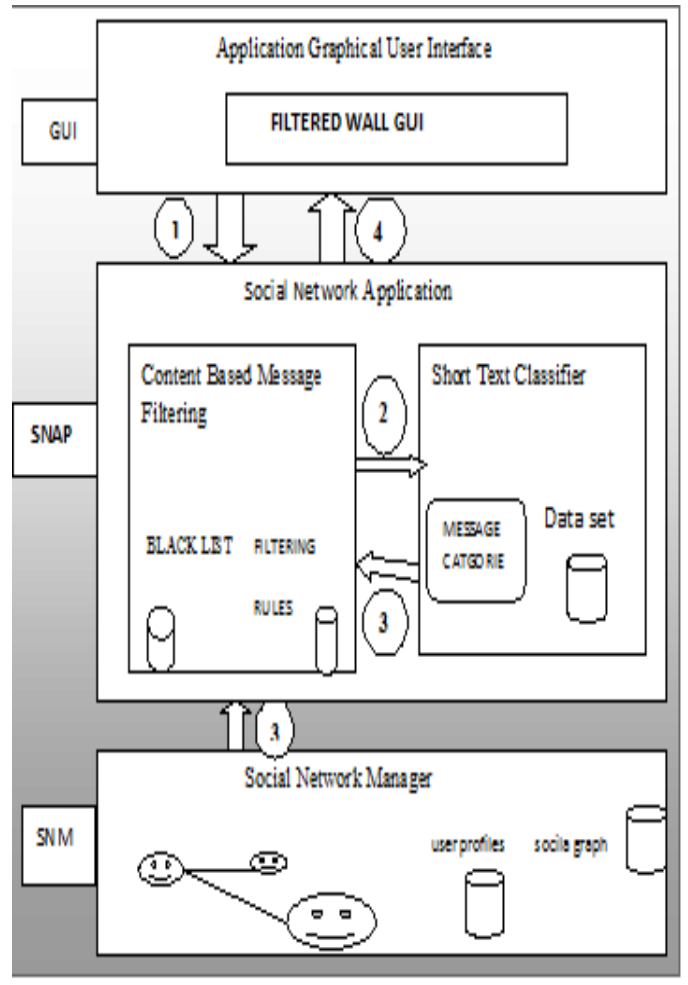

Fig1: Filtered Wall Architecture

The fundamental errand of Social system supervisor is to keep up the relationship and profile administration. It will keep up the information identified with the client profiles and the relationship in the middle of (FR) and blacklist (BL). Second layer made out of Content Based Message Filtering (CBMF) and short content order is a most critical layer. The classifier orders the every message as per its substance displayed in the message. The CBMF channels the message as per the separating criteria and the blacklist gave by the client. The bolstered SNAs might thus require an extra layer called the Graphical User Interface (GUIs). By references design, the proposed framework is set in the second and third layers. Specifically, the clients collaborate with the framework by method for GUI to set up and deal with their FLs/BLs.

\section{EXPERIMENTAL DESIGN}

Experimental design of the framework incorporates the whole information stream of our framework. Client once posts the message. Marks are characterized taking into account the substance. At that point the message will be separated. Likelihood of the message will be assessed. In light of that esteem the client will choose whether to embed in BL or not, GUI to set up and deal with their FLs/BLs. 


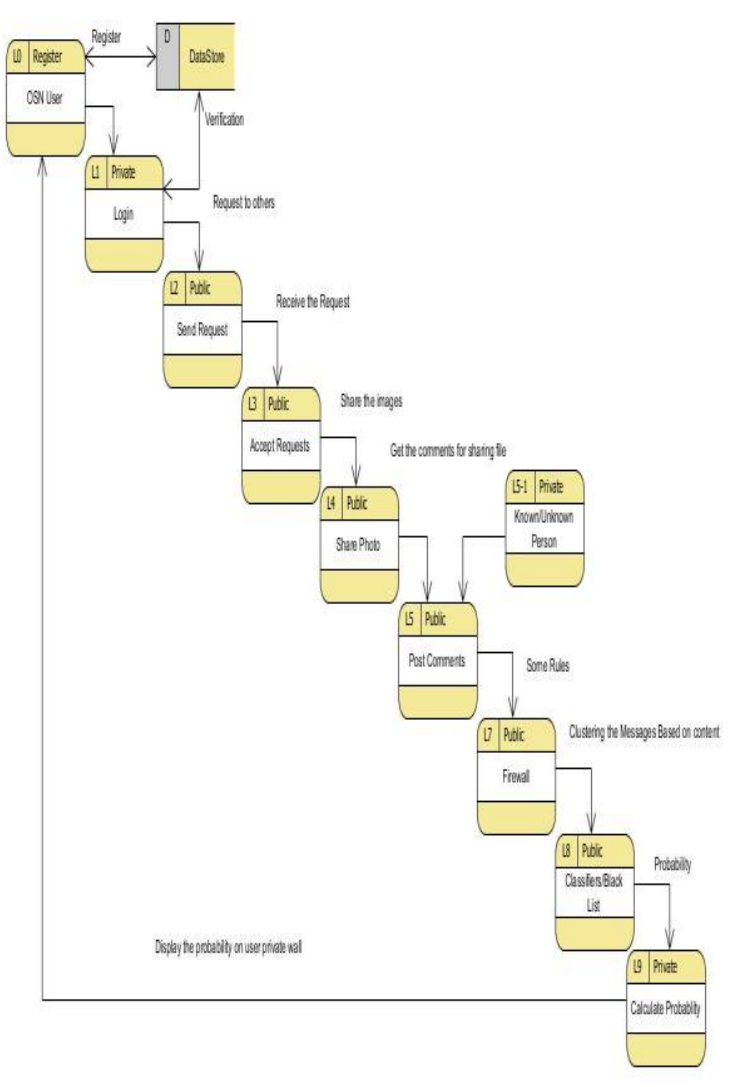

Fig: Experimental Diagram

\section{CONCLUSION}

In this paper, a framework to filter unwanted message in OSN divider is exhibited. The initial step of the undertaking is to group the substance utilizing a few standard. Next step is to channel the undesired principles. Blacklist tenet is actualized. At long last we led a huge scale estimation ponder that gathered information on the interpersonal organizations of four well known locales, covering more than 12 million clients and 400 million connections, keeping in mind the end goal to uproot the undesirable messages. So that proprietor of the client can embed the client who posts undesired messages. Better security is given to the OSN divider utilizing our framework. In future Work, we plan to execute the passing so as to separate rules with the point of the Sifting framework, it can be utilized just with the end goal of defeat the separating framework.

\section{REFERENCES}

[1] Marco Vanetti, Elisabetta Binaghi, Elena Ferrari, Barbara Carminati, an Moreno Carullo, " A System to Filter Unwanted Messages from OSN User Walls",2013.

[2] M.Chau and H.Chen," A Machine Learning Approach to Web Page Filtering Using Content and Structure Analysis," Decision Support Systems, vol.44, no.2, pp.482-494, 2008.

[3] F.Sebastiani, "Machine Learning Automated Text Categorization", ACM Computing surveys, vol.34, no.1, pp.1-47, 2002.

[4] B.Sriram, D.Fuhry, E.Demir, H.ferhatatosmanoglu, and M.Demirbas, "Short Text Classification in Twitter to Improve InformationFiltering," Proc.33rd Int'l ACM SIGIT Conf. Research and Development in Information Retrieval(sIGIR '10), pp.841-842,2010.

[5] V.Bobicev and M.Sokolova, "An Effective and Robust Method for Short Text Classification," Proc.23rd Nat'l Conf. Artificial Intelligence (AAAI), D.Fox and C.P.Gomes, eds., pp.1444-1445,2008.

[6] J.Colbeck, "Combining Provenance with Trust in Social Networks for Semantic Web Content Filtering," Proc. Int'l conf. Provenance and Annotation of Data, L.Moreau and I.Foster, eds., pp.101-108, 2006.

[7] M.Vanetti, E.Binaghi, B.Carminati, M.Carullo, and E.Ferrari, "Content- Based Filtering in On-Line Social Networks", 2010.

[8] M.Carullo, E.Binaghi, and I. Gallo, "An Online Document Clustering Technique for short Web contents, " Pattern Recognition Letters,vol.30, pp.870-876, July 2009.

[9] M.Carullo, E.Binaghi and I. Gallo, and N.Lamberti, "Clustering of Short commercial Documents for the web," Proc.19th Int'l conf. Pattern Recognition (ICPR '08), 2008.

[10] R.E.Schapire and Y.Singer, "Boostexter: A BoostingBased system for Text Categorization," Machine Learning, vol.39, nos.2/3, pp.135- 168, 2000.

[11] S.Zelikovitz and H.Hirsh, "Improving Short Text Classification Using Unlabeled Background Knowledge," Proc. 17th Int'l Conf. Machine Learning (ICML '00), P.Langley, ed.,pp.1183-1190, 2000.

[12] J.Nin, B.Carminati, E.Ferrari, and V.Torra, "Computing Reputation for Collaborative Private Networks," Proc.33rd Ann. IEEE Int'l computer Software and Applications Conf., Vol.1, pp. 246-253, 2009. 